\title{
Suppression of Parkinson's hand-like tremor using fuzzy-PID controller
}

\begin{abstract}
This paper presents the performance evaluation of an active vibration technique in suppressing human hand tremor. A PID integrated with fuzzy logic controller is proposed to control a linear electromagnet actuator and then applied onto a four Degree-of-Freedoms (DOFs) biodynamic model which emulate a Parkinson Disease (PD) hand. Heuristic method is employed in searching for appropriate parameters values for the proposed controller. Then, a comparative study between the performances of Fuzzy-PID controller and conventional PID controller tuned using Ziegler-Nichols method is done. The simulation study results demonstrates a comparable performance between Fuzzy-PID controller and conventional PID controller. Fuzzy-PID controller was effectively attenuated about $96.77 \%$ of initial tremor magnitude while for the PID controller is about $89.37 \%$.
\end{abstract}

Keyword: Active tremor control; Fuzzy logic; PID controller; Tremor 